\title{
Some Mathematical Facts About Optimal Cache Replacement
}

PIERRE MICHAUD, Inria

\begin{abstract}
This article exposes and proves some mathematical facts about optimal cache replacement that were previously unknown or not proved rigorously. An explicit formula is obtained, giving OPT hits and misses as a function of past references. Several mathematical facts are derived from this formula, including a proof that OPT miss curves are always convex, and a new algorithm called OPT tokens, for reasoning about optimal replacement.
\end{abstract}

CCS Concepts: $\bullet$ Computer systems organization $\rightarrow$ Superscalar architectures; $\bullet$ Theory of computation $\rightarrow$ Caching and paging algorithms;

Additional Key Words and Phrases: Cache, optimal replacement

ACM Reference Format:

Pierre Michaud. 2016. Some mathematical facts about optimal cache replacement. ACM Trans. Archit. Code Optim. 13, 4, Article 50 (December 2016), 19 pages.

DOI: http://dx.doi.org/10.1145/3017992

\section{INTRODUCTION}

Accessing a small and nearby memory is generally faster than accessing a large or distant one. Caching is the idea, ubiquitous in computer architecture, to keep some data in a small and nearby memory called a cache. The total dataset is often larger than the cache. When the requested data is not in the cache, this is a miss. Otherwise, this is a hit. Upon a miss, a decision must be made: should the accessed data be kept in the cache, and if so, which data should be evicted from the cache to make room for the missing data? This decision is taken by an algorithm called a replacement policy. Not all replacement policies generate the same number of misses. The performance and energy cost of a miss is greater than that of a hit, and we would like to have as few misses as possible.

Fifty years ago, Belady introduced a replacement policy minimizing the number of misses [Belady 1966]. However, this optimal policy requires to know future memory accesses, while replacement policies that can be implemented in practice generally base their decisions on past accesses. Still, optimal replacement is a valuable conceptual tool for evaluating and analyzing practical replacement policies. Even though practical replacement policies cannot be optimal in general, they can be optimal or close to optimal on certain classes of program behavior. Moreover, optimal replacement was a direct inspiration for some cache replacement policies, in principle implementable, that were proposed recently [Rajan and Govindarajan 2007; Jain and Lin 2016].

Although introduced five decades ago, optimal replacement is unintuitive and not completely understood. The main goal of this article is to expose new, unknown mathematical facts about optimal replacement and to prove them rigorously. In pursuing

Author's address: P. Michaud, Inria/IRISA, Campus de Beaulieu, 35042 RENNES Cedex; email: pierre. michaud@inria.fr.

Permission to make digital or hard copies of part or all of this work for personal or classroom use is granted without fee provided that copies are not made or distributed for profit or commercial advantage and that copies show this notice on the first page or initial screen of a display along with the full citation. Copyrights for components of this work owned by others than ACM must be honored. Abstracting with credit is permitted. To copy otherwise, to republish, to post on servers, to redistribute to lists, or to use any component of this work in other works requires prior specific permission and/or a fee. Permissions may be requested from Publications Dept., ACM, Inc., 2 Penn Plaza, Suite 701, New York, NY 10121-0701 USA, fax +1 (212) 869-0481, or permissions@acm.org.

(c) 2016 ACM 1544-3566/2016/12-ART50 $\$ 15.00$

DOI: http://dx.doi.org/10.1145/3017992 
this goal, we also clarify some facts about optimal replacement that we believe are not well known. We consider optimal replacement both with and without cache bypassing.

Although the term cache is used throughout this article, optimal replacement is of interest for any cache-like structure, such as Translation Lookaside Buffer (TLB), Branch Target Buffer (BTB), main memory (paging), etc. We call items the objects stored in the cache. For example, an item may be an aligned memory block (hardware cache), a virtual page address (TLB), a branch address (BTB), etc. In this study, we consider fully associative caches. Nevertheless, most of our conclusions apply to setassociative caches, as every cache set behaves like a small independent fully associative cache.

\section{STRUCTURE OF THE ARTICLE}

The goal of Sections 4 and 6 is to obtain Equations (14) and (18). All subsequent mathematical facts are derived from these equations. We provide some corroboration for these equations in Section 5 by proving the (already known) fact that all LRU hits are OPT hits, and in Section 7 by computing the OPT miss probability for random traces and comparing with Monte Carlo simulations. In Section 8, we introduce the OPT matrix, an algorithm for reasoning about all cache sizes simultaneously. Using the OPT matrix, we show that the output trace of OPT distances has a special structure, described by Theorem 1. Using Theorem 1, we prove in Section 9 that the OPT miss curve is always a convex function of the cache size, a fact discovered recently but not proved rigorously [Beckmann and Sanchez 2015]. In Section 10, we focus on a single cache size and derive from the OPT matrix an algorithm called the OPT tokens for obtaining OPT hits and misses. In Section 11, we derive directly from the OPT tokens an equivalence between a cache of size $j+1$ without bypassing and a cache of size $j$ with bypassing. Finally, in Section 12, and using the OPT tokens, we explain why accessing a dataset in a circular fashion maximizes the number of OPT misses. We cite related work where we think appropriate. Results for which we do not mention explicitly any related work are, to the best of our knowledge, new results.

This article contains only mathematical facts, independent of empirical properties of applications. We had to introduce a substantial amount of notations permitting to reason with precision. Our most important notations are listed in Table II.

\section{BELADY'S MIN AND MATTSON'S OPT}

Belady's 1966 paper [Belady 1966] and Mattson et al.'s 1970 paper [Mattson et al. 1970] are important and widely cited. Yet, there seems to be a long-lived confusion between Belady's MIN and Mattson's OPT. Many authors who referred to Belady's MIN actually described Mattson's OPT. ${ }^{1}$

Upon a cache miss, Mattson's OPT replacement policy evicts from the cache the item that will be re-referenced the furthest in time. This is stated very clearly by Mattson et al., who provide a proof that OPT is indeed optimal, in the sense that it minimizes the number of misses. Mattson et al. showed that OPT (like LRU) is a stack replacement policy, that is, the content of a cache of size $j$ is always included in a cache of size $j+1$. They introduced the OPT stack, based on the OPT replacement policy, for computing the minimum number of cache misses for all cache sizes at once.

Belady's MIN is not identical to Mattson's OPT. The MIN algorithm and the MIN replacement policy should be distinguished. The MIN algorithm is for determining hits and misses under optimal replacement [Belady 1966; Belady and Palermo 1974].

Table I shows how the MIN algorithm works on an example, assuming a 3-entry cache. MIN can be explained with a matrix where rows are associated with distinct

\footnotetext{
${ }^{1}$ The origin of this confusion is not clear. Confusion was already there in the early years [Aho et al. 1971; Coffman and Denning 1973].
} 
Table I. Belady's MIN Algorithm on an Example, Assuming a 3-Entry Cache. Each row of the MIN Matrix is Associated with a Distinct Item. Columns Represent Successive Times. Yellow Cells Show When Items are Referenced. A Cell is Marked " $r$ " When a Cache Entry is Reserved at that Time for that Item

\begin{tabular}{|c||c|c|c|c|c|c|c|c|c|c|c|c|c|c|c|c|c|c|}
\hline$t$ & 1 & 2 & 3 & 4 & 5 & 6 & 7 & 8 & 9 & 10 & 11 & 12 & 13 & 14 & 15 & 16 & 17 & 18 \\
\hline trace & $\mathrm{A}$ & $\mathrm{B}$ & $\mathrm{C}$ & $\mathrm{D}$ & $\mathrm{E}$ & $\mathrm{E}$ & $\mathrm{C}$ & $\mathrm{D}$ & $\mathrm{F}$ & $\mathrm{A}$ & $\mathrm{B}$ & $\mathrm{D}$ & $\mathrm{B}$ & $\mathrm{A}$ & $\mathrm{D}$ & $\mathrm{E}$ & $\mathrm{F}$ & $\mathrm{B}$ \\
\hline \hline \multicolumn{10}{|c|}{ MIN matrix } \\
\hline $\mathrm{9}$ & & $\mathrm{r}$ & & & & & & & & & $\mathrm{r}$ & $\mathrm{r}$ & $\mathrm{r}$ & $\mathrm{r}$ & $\mathrm{r}$ & & & \\
\hline $\mathrm{B}$ & & & $\mathrm{r}$ & & & & & & & & & $\mathrm{r}$ & $\mathrm{r}$ & $\mathrm{r}$ & $\mathrm{r}$ & $\mathrm{r}$ & $\mathrm{r}$ & $\mathrm{r}$ \\
\hline $\mathrm{C}$ & & & & $\mathrm{r}$ & $\mathrm{r}$ & $\mathrm{r}$ & $\mathrm{r}$ & $\mathrm{r}$ & & & & & & & & & & \\
\hline $\mathrm{D}$ & & & & & $\mathrm{r}$ & $\mathrm{r}$ & $\mathrm{r}$ & $\mathrm{r}$ & $\mathrm{r}$ & $\mathrm{r}$ & $\mathrm{r}$ & $\mathrm{r}$ & $\mathrm{r}$ & $\mathrm{r}$ & $\mathrm{r}$ & $\mathrm{r}$ & & \\
\hline $\mathrm{E}$ & & & & & & $\mathrm{r}$ & $\mathrm{r}$ & & & & & & & & & & $\mathrm{r}$ & \\
\hline $\mathrm{F}$ & & & & & & & & & & $\mathrm{r}$ & & & & & & & & $\mathrm{r}$ \\
\hline \hline hits & & & & & $\mathrm{h}$ & $\mathrm{h}$ & $\mathrm{h}$ & & & & $\mathrm{h}$ & $\mathrm{h}$ & $\mathrm{h}$ & $\mathrm{h}$ & & & $\mathrm{h}$ \\
\hline \hline
\end{tabular}

items and columns represent successive times. ${ }^{2}$ When the item referenced at time $t$ and previously referenced at time $t^{\prime}$ is determined to be in the cache at $t$ (hit), a cache entry is reserved for the item for the time interval $\left.] t^{\prime}, t+1\right]$, under the constraint that the number of reserved entries at any time cannot exceed the cache capacity. If cache entries cannot be reserved in $] t^{\prime}, t+1$ ] (because of the capacity constraint), this is a miss, and an entry is reserved only for the time $t+1$.

The MIN algorithm determines evicted items after a delay. More specifically, when a column of the MIN matrix contains a number of reservations equal to the cache capacity, we know which items are not in the cache and must have been evicted. For instance in Table I, at time $t=8$, the MIN algorithm determines that the cache contained items $\mathrm{C}, \mathrm{D}$, and $\mathrm{E}$ at time $t=7$, hence that items $\mathrm{A}$ and $\mathrm{B}$ had been evicted earlier. The MIN replacement policy attributes evictions to misses in the following way: among a group of items to evict, the least-recently referenced item is evicted the earliest [Belady 1966]. As can be seen in Table I, MIN and OPT, although both optimal, are different replacement policies. Note that, at $t=8$, when MIN determines the eviction of $\mathrm{A}$ and $\mathrm{B}$, it is not known yet which of $\mathrm{A}$ or $\mathrm{B}$ will be re-referenced first.

In 1966, Belady did not prove that MIN is optimal. The optimality of MIN was proved in 1974 when Belady and Palermo, relying on Mattson's proof of optimality for OPT, showed that MIN and OPT yield the same hits and misses [Belady and Palermo 1974].

Remarkably, the MIN algorithm is an online algorithm: it provides hit/miss information without delay, determining at time $t$, without look-ahead, if the item referenced at time $t$ is or is not in the cache. That is, even though optimal replacement depends on future references, whether the current reference is a hit or a miss depends only on past references. This property was not mentioned in the 1966 paper. It was emphasized later in the 1974 paper [Belady and Palermo 1974]. This property of MIN has been exploited in a new cache replacement policy published recently [Jain and Lin 2016].

\section{OPT HITS AND MISSES DEPEND ONLY ON PAST REFERENCES}

Belady's MIN algorithm demonstrates that, under optimal replacement, hits and misses depend only on past references. Hence, there must exist an explicit formula giving hits and misses as a function of past references. The goal of this section is to

\footnotetext{
${ }^{2}$ Belady's 1966 algorithm uses a vector, not a matrix. However, the matrix captures the essence of MIN and is easier to explain [Belady and Palermo 1974; Temam 1999; Jain and Lin 2016].
} 

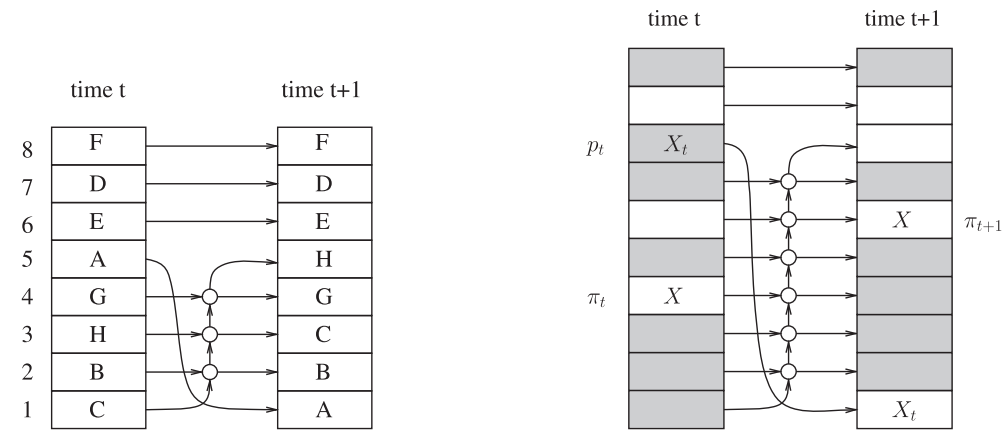

Fig. 1. Mattson's OPT stack on an example (no bypassing). Left: The item $X_{t}=A$ referenced at time $t$ is found at position $p_{t}=5$ in the stack. Circles represent sorting networks: the item whose time of next reference is furthest is sent to higher stack positions. The alphabetical order indicates how items are ordered at time $t$ by their time of next reference. Right: Another example, focusing on a particular item $X$. Darkened stack entries hold items that will be referenced before $X$. Item $X$ goes up in the stack when $X_{t}$ is above $X$ and all the stack positions below $X$ are occupied by items referenced before $X$.
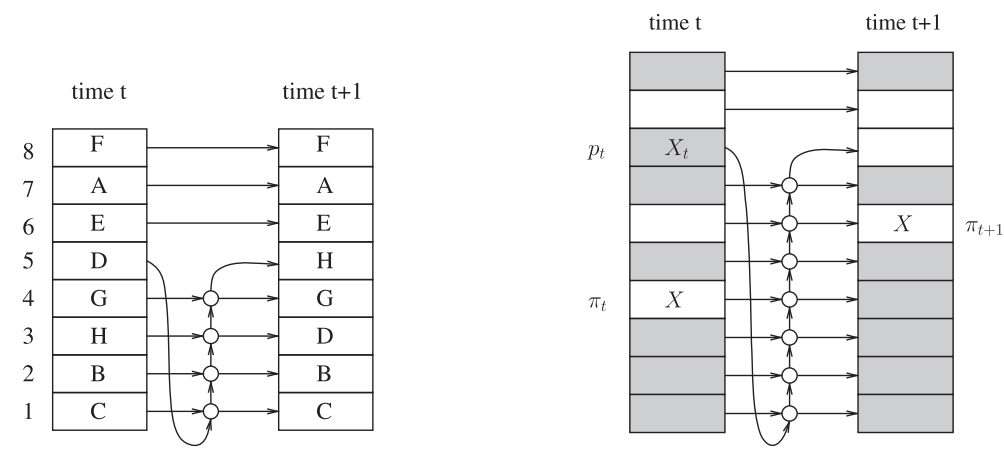

Fig. 2. OPT stack with bypassing. Left: The alphabetical order indicates how items are ordered at time $t+1$ by their time of next reference. Right: Focus on a particular item $X$. Item $X$ goes up in the stack when $X_{t}$ is above $X, X_{t}$ is re-referenced before $X$, and all the stack positions below $X$ are occupied by items that will be referenced before $X$.

find this formula (Equation (8)). Our motivation is that such formula will be useful for understanding OPT hits and misses.

Our starting point is the OPT replacement policy, for which multiple proofs of optimality are known [Mattson et al. 1970; Coffman and Denning 1973; Cohen and Burkhard 1995; Van Roy 2007; Vogler 2008; Lee et al. 2016].

Figure 1 (left part) illustrates Mattson's OPT-stack update procedure [Mattson et al. 1970]. In this example, the item A referenced at time $t$ in the reference stream is found at position 5 in the stack, meaning that 5 is the smallest cache size giving a cache hit at time $t$. Figure 2 gives a similar example where bypassing is allowed. In the article, we consider both the classical case (no bypassing) and the case with bypassing. We use parameter $\beta$ to indicate if bypassing is allowed $(\beta=1)$ or $\operatorname{not}(\beta=0)$.

Table II lists our main notations. We use the usual notations of set theory and the usual notations for intervals (e.g., $\left[t, t^{\prime}\left[\right.\right.$ is the set of values $\tau$ such that $t \leq \tau<t^{\prime}$ ). For $t<t^{\prime}, R_{\left[t, t^{\prime}[\right.}$ represents the set of items referenced at least once between times $t$ (included) and $t^{\prime}$ (not included). The stack size $s_{t}$ is the number of distinct items referenced at least once before time $t$, that is, $s_{t}=\left|R_{[0, t}\right|$. The OPT stack position of item $X_{t}$ referenced at time $t$ (aka OPT distance) is denoted $p_{t}$. If item $X_{t}$ is referenced 
Table II. Main Notations

\begin{tabular}{|c|c|c|c|}
\hline Notation & Definition & Description & Remark \\
\hline$\beta$ & \multicolumn{2}{|c|}{ bypassing is allowed $(\beta=1)$ or $\operatorname{not}(\beta=0)$} & $\beta \in\{0,1\}$ \\
\hline$R_{\left[t, t^{\prime}[\right.}$ & \multicolumn{2}{|c|}{ set of items referenced in the time interval $\left[t, t^{\prime}[\right.$} & $R_{[t, t[}=\varnothing$ \\
\hline$X_{t}$ & \multicolumn{2}{|c|}{ item referenced at time $t$} & $\left\{X_{t}\right\}=R_{[t, t+1[}$ \\
\hline$s_{t}$ & $s_{t}:=\left|R_{[0, t[}\right|$ & $\begin{array}{l}\text { number of distinct items } \\
\text { referenced before time } t\end{array}$ & \\
\hline$C_{t}^{j}$ & \multicolumn{2}{|c|}{ set of items in an OPT cache of size $j$ at time $t$} & $C_{t}^{0}=\varnothing, C_{t}^{s t}=R_{[0, t[}$ \\
\hline$p_{t}$ & \multicolumn{2}{|c|}{ OPT stack position of item $X_{t}$} & $\left\{X_{t}\right\}=C_{t}^{p_{t}}-C_{t}^{p_{t}-1}$ \\
\hline$M_{\left[t^{\prime}, t[\right.}^{j}$ & $M_{\left[t^{\prime}, t[\right.}^{j}:=\mid\left\{\tau \in\left[t^{\prime}, t\left[: p_{\tau}>j\right\} \mid\right.\right.$ & $\begin{array}{c}\text { number of misses for the } \\
\text { cache of size } j \text { in the } \\
\text { time interval }\left[t^{\prime}, t[\right.\end{array}$ & $M_{\left[t^{\prime}, t[\right.}^{s t}=0$ \\
\hline$u_{t}(i)$ & $u_{t}(i):=\max \left\{\tau \leq t:\left|R_{[\tau, t[}\right|=i\right\}$ & $\begin{array}{l}\text { greatest time } \tau \text { such } \\
\text { that } i \text { distinct items are } \\
\text { referenced in }[\tau, t[\end{array}$ & $u_{t}(1)=t-1, u_{t}(i+1)<u_{t}(i)$ \\
\hline$q_{t}$ & $q_{t}:=\min \left\{i \geq 1: X_{u_{t}(i)}=X_{t}\right\}$ & $\begin{array}{c}\text { LRU stack position of } \\
\text { item } X_{t}\end{array}$ & $X_{t} \notin R_{\left[u_{t}\left(q_{t}\right)+1, t[\right.}$ \\
\hline$D_{t}^{j}(i)$ & $D_{t}^{j}(i):=i-M_{\left[u_{t}(i)-\beta+1, t[\right.}^{j}$ & & $D_{t}^{s_{t}}(i)=i$ \\
\hline$I_{t}^{j}$ & $I_{t}^{j}:=\min \left\{i \in\left[1, s_{t}\right]: D_{t}^{j}(i)=j\right\}$ & & $I_{t}^{s t}=s_{t}$ \\
\hline
\end{tabular}

for the first time, we define $p_{t}=s_{t}+1=s_{t+1} \cdot C_{t}^{j}$ is the set of items in a cache of size $j$ (items) at time $t$ under optimal replacement $\left(C_{t}^{0}=\varnothing\right)$. That is, $C_{t}^{j}$ is the set of items whose position in the OPT stack at $t$ does not exceed position $j$.

Let us focus on one item, $X$, referenced at time $t_{2}$, and let us assume that $X$ was previously referenced at time $t_{1}<t_{2}-1$. That is, $X=X_{t_{1}}=X_{t_{2}}$ and $X \notin R_{\left[t_{1}+1, t_{2}[\right.}$.

Let $\pi_{t} \in\left[1, s_{t}\right]$ denote the position of $X$ in the OPT stack at time $\left.\left.t \in\right] t_{1}, t_{2}\right]$. First, it should be observed that the stack position of an item cannot decrease unless the item is referenced. As $X$ is not referenced during $] t_{1}, t_{2}\left[, \pi_{t}\right.$ is nondecreasing in $\left.] t_{1}, t_{2}\right]$. A necessary condition for $X$ to go up in the stack (i.e., $\pi_{t+1}>\pi_{t}$ ) is that $p_{t}>\pi_{t}$ and all the stack positions below $\pi_{t}$ are occupied by items that are referenced in $\left[t+1, t_{2}[\right.$. A useful quantity to consider is the number $N_{t}^{j}$ of items in the cache of size $j$, at time $t$, that are referenced in $\left[t, t_{2}[\right.$ :

$$
\text { for } \left.t \in] t_{1}, t_{2}\right] \quad N_{t}^{j}:=\left|C_{t}^{j} \cap R_{\left[t, t_{2}[\right.}\right| \text {. }
$$

Another useful quantity is

$$
e_{t}:=\left|R_{\left[t, t_{2}[\right.}\right|-\left|R_{\left[t+1, t_{2}[\right.}\right|
$$

Notice that $e_{t} \in\{0,1\}$ and

$$
N_{t+1}^{j}+(1-\beta) e_{t} \leq j .
$$

When $X$ goes up in the stack, the new position of $X$ is $h_{t+1}$ defined as

$$
h_{t+1}:=\min \left\{j \in\left[1, s_{t+1}\right]: N_{t+1}^{j}<j-(1-\beta) e_{t}\right\} .
$$

For $t \in] t_{1}, t_{2}$ [, we have $\pi_{t+1}=\max \left(\pi_{t}, h_{t+1}\right)$. Note that $\pi_{t_{1}+1}=1$ for $\beta=0$ and $\pi_{t_{1}+1}=$ $h_{t_{1}+1}$ for $\beta=1$. Therefore,

$$
\left.\left.p_{t_{2}}=\pi_{t_{2}}=\max \left\{h_{t}: t \in\right] t_{1}+1-\beta, t_{2}\right]\right\} .
$$


The OPT stack update procedure leads to the following recurrence relation:

$$
\begin{array}{ll}
j \geq p_{t}, & N_{t+1}^{j}=N_{t}^{j}-e_{t}, \\
j<p_{t}, & N_{t+1}^{j}=\min \left(j, N_{t}^{j}+1-\beta e_{t}\right)-(1-\beta) e_{t} .
\end{array}
$$

The values $N_{t_{1}+1}^{j}$ are not known. However, we know that $N_{t_{2}}^{j}=0$. If recurrence (3) could be reversed, we could obtain $N_{t}^{j}$. Actually, recurrence (3) can be reversed partially. Notice that $j<p_{t}$ means a miss in the cache of size $j$ at time $t$. Let us define

$$
M_{\left[t^{\prime}, t[\right.}^{j}:=\mid\left\{\tau \in \left[t^{\prime}, t\left[: p_{\tau}>j\right\} \mid .\right.\right.
$$

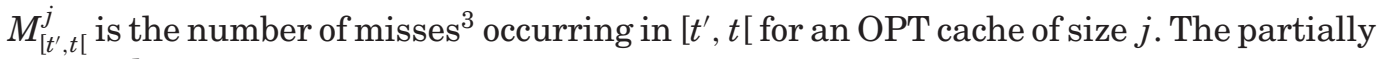
reversed recurrence is

$$
N_{t+1}^{j}<j-(1-\beta) e_{t} \quad \Longrightarrow \quad N_{t}^{j}-N_{t+1}^{j}=e_{t}-M_{[t, t+1[\cdot}^{j}
$$

Let us denote $\operatorname{rev}(t)$ the condition on the left-hand side of (4):

$$
\operatorname{rev}(t): \quad N_{t+1}^{j}<j-(1-\beta) e_{t} .
$$

We have

$$
\operatorname{rev}(\tau) \forall \tau \in\left[t, t_{2}\left[\Longrightarrow N_{t}^{j}-N_{t_{2}}^{j}=\sum_{\tau \in\left[t, t_{2}[\right.}\left(e_{\tau}-M_{[\tau, \tau+1]}^{j}\right) .\right.\right.
$$

Hence,

$$
\operatorname{rev}(\tau) \forall \tau \in\left[t, t_{2}\left[\quad \Longrightarrow \quad N_{t}^{j}=\left|R_{\left[t, t_{2}[\right.}\right|-M_{\left[t, t_{2}[\right.}^{j}\right.\right.
$$

and

$$
\operatorname{rev}(\tau) \forall \tau \in\left[t+1, t_{2}\left[\quad \Longrightarrow \quad N_{t+1}^{j}+(1-\beta) e_{t}=\left|R_{\left[t+\beta, t_{2}[\right.}\right|-M_{\left[t+1, t_{2}[\right.}^{j} .\right.\right.
$$

As the equality on the right-hand side of (5) is always true for $t=t_{2}-1$, we can apply a backward induction on condition $\operatorname{rev}(\tau)$ :

$$
\operatorname{rev}(\tau) \forall \tau \in\left[t, t_{2}\left[\Longleftrightarrow \forall \tau \in \left[t, t_{2}\left[\quad\left|R_{\left[\tau+\beta, t_{2}[\right.}\right|-M_{\left[\tau+1, t_{2}[\right.}^{j}<j .\right.\right.\right.\right.
$$

Let us define for $\beta=0, j>1$ and for $\beta=1, j>0$

$$
T_{t_{2}}^{j}:=\min \left\{t<t_{2}: \forall \tau \in\left[t, t_{2}\left[,\left|R_{\left[\tau+\beta, t_{2}[\right.}\right|-M_{\left[\tau+1, t_{2}[\right.}^{j}<j\right\}\right.\right.
$$

and for $\beta=0, T_{t_{2}}^{1}:=t_{2}$.

Consider the case $T_{t_{2}}^{j}>t_{1}+1-\beta$. Then for $t=T_{t_{2}}^{j}-1>t_{1}-\beta$, by definition (7) and using equivalence (6), condition rev(t) must be false. Hence, $h_{t+1}>j$, which implies (from (2)) $p_{t_{2}}>j$. On the other hand, when $T_{t_{2}}^{j} \leq t_{1}+1-\beta$, condition $\operatorname{rev}(t)$ is true for all $t \in\left[t_{1}+1-\beta, t_{2}\left[\right.\right.$, hence $h_{t+1} \leq j$ for all $t \in\left[t_{1}+1-\beta, t_{2}\left[\right.\right.$, and $p_{t_{2}} \leq j$. Therefore,

$$
p_{t_{2}}=\min \left\{j \geq 1: T_{t_{2}}^{j} \leq t_{1}+1-\beta\right\} \text {. }
$$

So far, we have assumed $t_{2}>t_{1}+1$, but Equation (8) is valid also for $t_{2}=t_{1}+1\left(p_{t_{2}}=1\right)$. It should be noted that $T_{t_{2}}^{j}$ does not depend on which item is referenced at $t_{2}$. Only the $t_{1}$ in Equation (8) depends on the item referenced at $t_{2}$.

\footnotetext{
${ }^{3}$ We recall that when item $X_{\tau}$ is referenced for the first time at $\tau \in\left[t^{\prime}, t\right.$, we define $p_{\tau}=s_{\tau}+1$. With our definition of $M_{\left[t^{\prime}, t\right.}^{j}$, the reference at $\tau$ is counted as a hit for all $j \geq s_{\tau}+1$.
} 
We end this section with two facts that are used later in the article. First,

$$
\forall j \quad T_{t}^{j+1} \leq T_{t}^{j}
$$

This can be seen as follows. Instead of referencing $X_{t}$ at $t=t_{2}$, let us reference the item that was last referenced at $t_{1}=T_{t}^{j}-1+\beta$. That item exists because, by definition of $T_{t}^{j},\left|R_{[\tau+\beta, t}\right|$ must be incremented when going from $\tau=T_{t}^{j}$ to $\tau=T_{t}^{j}-1$. As $T_{t}^{j}=t_{1}+1-\beta$, we must have $p_{t} \leq j$, hence $p_{t} \leq j+1$, which implies $T_{t}^{j+1} \leq t_{1}+1-\beta$, that is, $T_{t}^{j+1} \leq T_{t}^{j}$.

The second fact is

$$
\tau \in\left[T_{t}^{j}, t\left[\Longrightarrow M_{[\tau, t[}^{j}-M_{[\tau, t[}^{j+1} \in\{0,1\} .\right.\right.
$$

To see this, let us reference at $t=t_{2}$ the item that was last referenced at $t_{1}=T_{t}^{s_{t}}-1+\beta$, and let us consider $j<s_{t}$. We must have $T_{t}^{j+1} \geq T_{t}^{s_{t}}=t_{1}+1-\beta$. Hence, for $\tau \in\left[T_{t}^{j}, t\right]$, quantities $N_{\tau}^{j}$ and $N_{\tau}^{j+1}$ are defined. From (7), (6), and (5), we have

$$
\begin{aligned}
\tau \in\left[T_{t}^{j}-1, t[\right. & \Longrightarrow \operatorname{rev}\left(t^{\prime}\right) \quad \forall t^{\prime} \in[\tau+1, t[ \\
& \Longrightarrow N_{\tau+1}^{j}+(1-\beta) e_{\tau}=\left|R_{[\tau+\beta, t[}\right|-M_{[\tau+1, t[}^{j} \\
& \Longrightarrow \quad M_{[\tau+1, t[}^{j}-M_{[\tau+1, t[}^{j+1}=N_{\tau+1}^{j+1}-N_{\tau+1}^{j}
\end{aligned}
$$

which, from definition (1), proves (10).

\section{ALL LRU HITS ARE OPT HITS}

The fact that OPT is optimal means that no replacement policy can generate fewer misses than OPT. However, an individual reference that is a hit under a given policy $\mathrm{P}$ is not necessarily a hit under OPT. Still, it is known (though perhaps not widely) that every LRU hit is also an OPT hit [Puzak 1985; Lin and Reinhardt 2002; Brock et al. 2013]. It can be verified that Equation (8) is consistent with this fact. Let us assume $p_{t_{2}}>1$. From (8), we must have $T_{t_{2}}^{p_{t_{2}}-1}>t_{1}+1-\beta$. Letting $t=T_{t_{2}}^{p_{t_{2}}-1}-1$, and from $(7)$,

$$
\begin{aligned}
p_{t_{2}}-1 & =\left|R_{\left[t+\beta, t_{2}[\right.}\right|-M_{\left[t+1, t_{2}[\right.}^{p_{t_{2}}-1} \\
& \leq\left|R_{\left[t+\beta, t_{2}[\right.}\right| \\
& <\left|R_{\left[t_{1}, t_{2}[\right.}\right| .
\end{aligned}
$$

Let us define

$$
q_{t_{2}}:=\left|R_{\left[t_{1}, t_{2}\right.}\right| \text {. }
$$

Quantity $q_{t_{2}}$ is the LRU stack position (aka reuse distance) of the item $X$ referenced at time $t_{2}$. We have

$$
p_{t_{2}} \leq q_{t_{2}} .
$$

Inequation (11) is valid in the case $p_{t_{2}}=1$, and also when item $X_{t_{2}}$ is referenced for the first time ( $\left.p_{t_{2}}=q_{t_{2}}=\left|R_{\left[0, t_{2}\right.}\right|+1\right)$. In words, inequation (11) means that every LRU hit is also an OPT hit.

There is something remarkable here. The LRU replacement policy, which evicts from the cache the item referenced the least recently, is an online policy: it makes replacement decisions without knowing future references. LRU is not optimal in general, but it works well in many practical applications. The performance of LRU lies in empirical 
properties of traces, not in LRU itself. Indeed, if we consider random traces (items have equal probability to be referenced), all online replacement policies yield the same average hit ratio, equal to the cache size divided by the number of distinct items. Without considering trace properties, LRU is neither better nor worse than any other online policy. Still, inequation (11) highlights a special relationship between LRU and OPT. This suggests a (rather theoretical) question, which we will not try to answer in this article: Is there an online policy $\mathrm{P}$ other than LRU such that, for any trace, all hits under policy $\mathrm{P}$ are hits under OPT?

\section{PUTTING THE REUSE DISTANCE INTO THE EQUATION}

Equation (8) is written in terms of $t_{1}$ and $t_{2}$. In this section, we rewrite it in terms of $t$ and $q_{t}$ (the reuse distance). Let us define

$$
I_{t}^{j}:=\left|R_{\left[T_{t}^{j}-1+\beta, t[\right.}\right| .
$$

Note that (9) implies

$$
\forall j \quad I_{t}^{j+1} \geq I_{t}^{j}
$$

Equation (8) can be written

$$
p_{t}=\min \left\{j \geq 1: I_{t}^{j} \geq q_{t}\right\}
$$

except for the special case $p_{t}=s_{t}+1$ for a first reference. As we defined it, $I_{t}^{j}$ depends on $T_{t}^{j}$. For the sake of convenience, we would like to find a more direct expression for $I_{t}^{j}$. Let us introduce the quantity

$$
D_{[\tau, t[}^{j}:=\left|R_{[\tau+\beta, t[}\right|-M_{[\tau+1, t[}^{j} .
$$

We can rewrite (7) as

$$
T_{t}^{j}=\min \left\{t^{\prime}<t: \forall \tau \in\left[t^{\prime}, t\left[, D_{[\tau, t[}^{j}<j\right\} .\right.\right.
$$

When $\tau$ decreases, $D_{[\tau, t[}^{j}$ increases only when $\left|R_{[\tau+\beta, t[}\right|$ is incremented. Instead of considering all values $\tau$, we can focus on the values $\tau$ such that $\left|R_{[\tau, t}\right|$ is incremented. For $i>0$, let

$$
u_{t}(i):=\max \left\{\tau<t:\left|R_{[\tau, t \mid}\right|=i\right\} .
$$

As $\left|R_{\left[T_{t}^{j}+\beta, t\right.}\right|<I_{t}^{j}$ (cf. the argument used in proving (9)), we must have

$$
u_{t}\left(I_{t}^{j}\right)=T_{t}^{j}-1+\beta .
$$

Consequently,

$$
\begin{aligned}
i<I_{t}^{j} & \Longrightarrow \quad u_{t}(i)>u_{t}\left(I_{t}^{j}\right) \\
& \Longrightarrow \quad u_{t}(i)-\beta \geq T_{t}^{j} \\
& \Longrightarrow \quad D_{\left[u_{t}(i)-\beta, t[\right.}^{j}<j
\end{aligned}
$$

Moreover,

$$
D_{\left[u_{t}\left(I_{t}^{j}\right)-\beta, t[\right.}^{j}=D_{\left[T_{t}^{j}-1, t[\right.}^{j}=j .
$$

Let us define for $i \in\left[1, s_{t}\right]$

$$
D_{t}^{j}(i):=D_{\left[u_{t}(i)-\beta, t[\right.}^{j}=i-M_{\left[u_{t}(i)-\beta+1, t[\right.}^{j} .
$$


Table III. Example: Determining hit/miss at time $t$ from Past hits/Misses $(\beta=1$ and Cache Size $j=3)$

\begin{tabular}{|c||c|c|c|c|c|c|c|c|c|c|c|}
\hline time & $\cdots$ & $t-9$ & $t-8$ & $t-7$ & $t-6$ & $t-5$ & $t-4$ & $t-3$ & $t-2$ & $t-1$ & $t$ \\
\hline trace & $\cdots$ & $\mathrm{A}$ & $\mathrm{D}$ & $\mathrm{F}$ & $\mathrm{C}$ & $\mathrm{F}$ & $\mathrm{E}$ & $\mathrm{C}$ & $\mathrm{B}$ & $\mathrm{B}$ & $\mathrm{A}$ \\
\hline hit/miss & $\cdots$ & & hit & miss & hit & hit & miss & hit & miss & hit & hit \\
\hline$u_{t}(i)$ & $\cdots$ & $u_{t}\left(q_{t}\right)$ & $u_{t}(5)$ & & & $u_{t}(4)$ & $u_{t}(3)$ & $u_{t}(2)$ & & $u_{t}(1)$ & \\
\hline$M_{\left[u_{t}(i), t\right.}^{3}$ & $\cdots$ & & 3 & & & 2 & 2 & 1 & & 0 & \\
\hline$D_{t}^{3}(i)$ & $\cdots$ & & 2 & & & 2 & 1 & 1 & & 1 & \\
\hline
\end{tabular}

We find that $I_{t}^{j}$ can be obtained as

$$
I_{t}^{j}=\min \left\{i \in\left[1, s_{t}\right]: D_{t}^{j}(i)=j\right\} .
$$

Notice that $D_{t}^{j}(i) \leq i$, hence from (18), $j=D_{t}^{j}\left(I_{t}^{j}\right) \leq I_{t}^{j}$. Therefore, $I_{t}^{q_{t}} \geq q_{t}$ and, from (14), $p_{t} \leq q_{t}$ (already shown in Section 5). The rest of the article is built on Equations (14) and (18).

If we focus on a single cache size $j$, the procedure for determining whether the reference at time $t$ is a hit or a miss is simply:

$$
\text { OPT hit at time } \begin{aligned}
& \Longleftrightarrow p_{t} \leq j \\
& \Longleftrightarrow I_{t}^{j} \geq q_{t} \\
& \Longleftrightarrow \quad \forall i<q_{t}, D_{t}^{j}(i)<j .
\end{aligned}
$$

This is illustrated in Table III on an example, assuming bypassing $(\beta=1)$ and cache size $j=3$. This example shows how the hit/miss outcome at time $t$ is obtained from past hit/miss outcomes. On this example, this is a hit at time $t$, as all the $D_{t}^{j}(i)$ values for $i<q_{t}$ are strictly less than three.

\section{RANDOM TRACES}

Equations (14) and (18) can be used to compute the OPT miss probability for random traces, that is, traces such that the number $s$ of distinct items is fixed and items have equal probability 1/s to be referenced. Smith [1976] and Knuth [1985] have shown that the exact miss ratio of an OPT cache of size $j$ on random traces can be obtained by solving a Markov chain with $\left(\begin{array}{c}s \\ j\end{array}\right)$ states. According to Knuth, there does not seem to be any simple formula for the exact OPT miss ratio of random traces for any $s$ and $j$ in general, although Knuth found closed-form expressions for the special cases $j=2$ and $j=s-1$. In general, for obtaining the OPT miss ratio on random traces, one must solve a (possibly huge) Markov chain or do Monte Carlo simulations.

Here we search an approximation for the case $s \rightarrow \infty, j \rightarrow \infty$ but $j / s \in[0,1]$ fixed. Let us consider an interval $[t, t+\Delta t$ [ with $\Delta t$ large. For a given item $x$, the probability that $x$ is not in $R_{[t, t+\Delta t[}$ is $\left(1-\frac{1}{s}\right)^{\Delta t}$. Hence,

$$
\begin{aligned}
\left|R_{[t, t+\Delta t \mid}\right| & \approx s \times\left(1-\left(1-\frac{1}{s}\right)^{\Delta t}\right) \\
& \approx s \times\left(1-e^{-\frac{\Delta t}{s}}\right) .
\end{aligned}
$$

Then, we have

$$
t-u_{t}(i) \approx-s \times \ln \left(1-\frac{i}{s}\right)
$$

Denoting $m$ the miss probability and taking $\beta=1$, we have

$$
D_{t}^{j}(i) \approx i+s \times \ln \left(1-\frac{i}{s}\right) \times m
$$




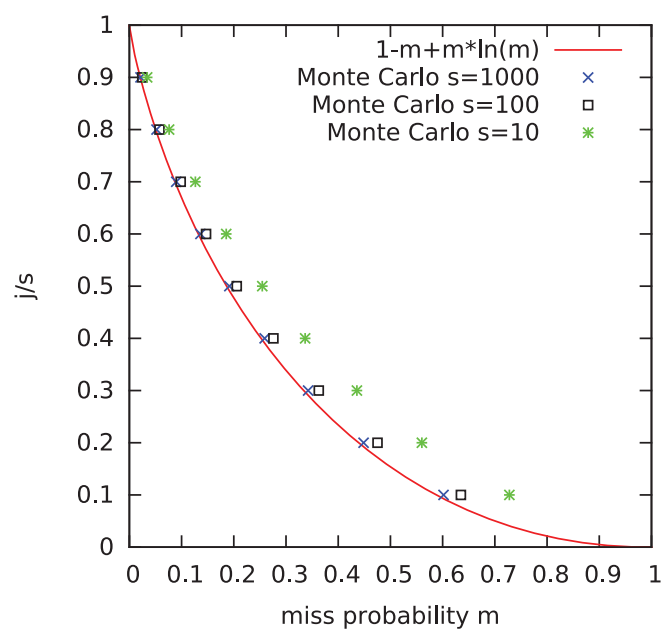

Fig. 3. OPT cache of size $j$ (bypassing allowed) and random trace with $s$ distinct items. Relation between $j / s$ and the miss probability $m$.

As $D_{t}^{j}\left(I_{t}^{j}\right)=j$, we obtain the following equation:

$$
j \approx I_{t}^{j}+s \times \ln \left(1-\frac{I_{t}^{j}}{s}\right) \times m .
$$

But $m$ is the probability that $p_{t}>j$, that is, the probability that $q_{t}>I_{t}^{j}$. As $q_{t}$ is uniformly distributed in $[1, s]$, we have

$$
m=1-\frac{I_{t}^{j}}{s}
$$

Substituting $\frac{I_{t}^{j}}{s}$ with $1-m$, we obtain

$$
\frac{j}{s} \approx 1-m+m \times \ln (m)
$$

Figure 3 shows a plot of $j / s$ versus $m$ as given by (19), compared with Monte Carlo simulations. Formula (19) models the asymptotic behavior and is more accurate as $s$ gets larger. OPT is very effective on random traces: asymptotically, the number of distinct items must be about 10 times the cache size for the OPT miss ratio to exceed $60 \%$.

\section{THE OPT MATRIX}

In Section 6, we considered a fixed $t$. Let us look at how $D_{t}^{j}(i)$ changes from $t$ to $t+1$. First, notice that

$$
\begin{array}{ll}
i \leq q_{t}, & u_{t+1}(i)=u_{t}(i-1), \\
i>q_{t}, & u_{t+1}(i)=u_{t}(i) .
\end{array}
$$

Then, from (17), we obtain the following update procedure for $p_{t} \in\left[1, s_{t}\right]$ :

$$
\begin{array}{|c|l|l|l|}
\cline { 2 - 4 } \multicolumn{1}{c|}{} & \multicolumn{1}{c|}{i>q_{t}} & \multicolumn{1}{c|}{i \in\left[2, q_{t}\right]} & \multicolumn{1}{c|}{i=1} \\
\hline j \in\left[p_{t}, s_{t}\right] & D_{t+1}^{j}(i)=D_{t}^{j}(i) & D_{t+1}^{j}(i)=D_{t}^{j}(i-1)+1 & D_{t+1}^{j}(1)=1 \\
\hline j<p_{t} & D_{t+1}^{j}(i)=D_{t}^{j}(i)-1 & D_{t+1}^{j}(i)=D_{t}^{j}(i-1) & D_{t+1}^{j}(1)=1-\beta \\
\hline
\end{array}
$$


In the special case $q_{t}=p_{t}=s_{t}+1=s_{t+1}$, we have $D_{t+1}^{s_{t}+1}(i)=i$. Procedure (20) (along with Equations (14) and (18)) defines an algorithm for computing $p_{t}$. We call this algorithm the OPT matrix. The OPT matrix is not intended for analyzing real traces. It is inefficient in computational complexity and memory usage. ${ }^{4}$ Nevertheless, the OPT matrix is a useful conceptual tool for reasoning about OPT, considering all cache sizes simultaneously. In particular, we are going to show that, whatever the input trace, the sequence of OPT distances has a remarkable structure.

Theorem 1. Given $t^{\prime}>t$ such that $p_{t^{\prime}}=p_{t}>2-\beta$ and $\left.\forall \tau \in\right] t, t^{\prime}\left[, p_{\tau} \neq p_{t}, \forall j \in\right.$ $\left[2-\beta, p_{t}[\right.$ there exists $\tau \in] t, t^{\prime}\left[\right.$ such that $p_{\tau}=j$.

In words, Theorem 1 states that, in the sequence of OPT distances $p_{t}$, a given distance greater than $2-\beta$ cannot reoccur until all lower distances (except 1 in the no-bypassing case) have occurred at least once. Next is a sequence of OPT distances that we generated from a random input trace with eight distinct items, without bypassing:

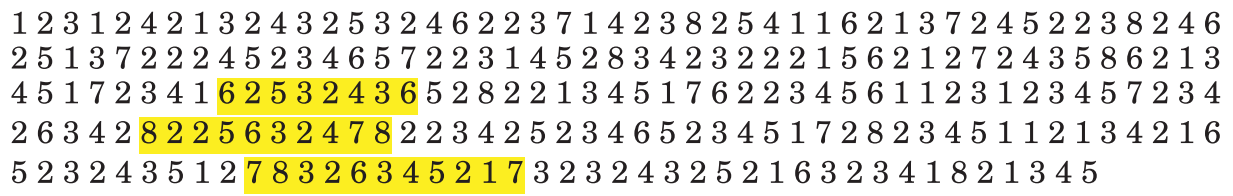

We have highlighted a few parts of the sequence to illustrate Theorem 1 . Here is the sequence of OPT distances produced from the same input trace when bypassing is allowed:

\section{7 14128312451326471231452813213121452126173482113 54162341723125346181213451762134561123123457123 16243812354167821341521364152317281324112134115 612341512783162453172132413511621341821342}

Notice that, without bypassing, distance 2 can occur several times consecutively, while this does not occur with bypassing.

Before proving Theorem 1, let us make some preliminary observations. From (17) and (10) we must have

and for any $k \geq 0$

$$
\forall i \in\left[1, I_{t}^{j}\right], \quad D_{t}^{j+1}(i)-D_{t}^{j}(i) \in\{0,1\}
$$

$$
\forall i \in\left[1, I_{t}^{j}\right], \quad D_{t}^{j+k}(i) \leq D_{t}^{j}(i)+k
$$

Also, observe (from (17)) that

$$
D_{t}^{j}(i) \neq D_{t}^{j-1}(i) \quad \Longrightarrow \quad D_{t}^{j}(i+1) \neq D_{t}^{j-1}(i+1) .
$$

Let us define

$$
Y_{t}^{j}:=\min \left\{i \geq 1: D_{t}^{j}(i) \neq D_{t}^{j-1}(i)\right\}
$$

As $D_{t}^{j-1}\left(I_{t}^{j-1}\right)=j-1$,

$$
I_{t}^{j}>I_{t}^{j-1} \quad \Longrightarrow \quad D_{t}^{j}\left(I_{t}^{j-1}\right)<j \quad \Longrightarrow \quad D_{t}^{j}\left(I_{t}^{j-1}\right)=j-1 \quad \Longrightarrow \quad Y_{t}^{j}>I_{t}^{j-1} \text {. }
$$

\footnotetext{
${ }^{4}$ Our goal here is not to obtain a computationally efficient algorithm. Sugumar and Abraham described an efficient OPT stack implementation requiring a single pass (unlike Mattson's original algorithm), but with some look-ahead [Sugumar and Abraham 1993]. They implemented this algorithm in a cache simulator called Cheetah, distributed with the Simplescalar processor simulation infrastructure [Burger and Austin 1997]. We believe that Cheetah is fast enough for most practical purposes.
} 
On the one hand, from (13), (14), (18), and (20), we have

$$
\forall j, \quad I_{t}^{j}<I_{t}^{p_{t}} \Longrightarrow I_{t+1}^{j}=I_{t}^{j}+1 \text {. }
$$

On the other hand, consider the smallest $j$ such that $I_{t}^{j}>I_{t}^{p_{t}}$. As $I_{t}^{j}>I_{t}^{j-1}=I_{t}^{p_{t}}$, we have $Y_{t}^{j}>I_{t}^{j-1}=I_{t}^{p_{t}}$, hence $D_{t}^{j}(i)=D_{t}^{j-1}(i)$ for all $i \leq I_{t}^{p_{t}}$. Therefore,

$$
i<I_{t}^{p_{t}} \Longrightarrow D_{t}^{j-1}(i)<j-1 \quad \Longrightarrow \quad D_{t}^{j}(i)<j-1 \quad \Longrightarrow \quad D_{t}^{j+k}(i)<j+k-1
$$

for any $k \geq 0$. Consequently, and from (20),

$$
\forall j, \quad I_{t}^{j}>I_{t}^{p_{t}} \Longrightarrow I_{t+1}^{j}=I_{t}^{j} .
$$

$I_{t}^{j}$ cannot decrease unless $I_{t}^{j}=I_{t}^{p_{t}}$. From (20), we must have

$$
\forall j, \quad I_{t}^{j}=I_{t}^{j-1}=I_{t}^{p_{t}} \quad \Longrightarrow \quad I_{t+1}^{j} \geq Y_{t}^{j}+1
$$

From (20), $Y_{t}^{j}$ is updated as follows:

$$
\begin{array}{lll} 
& & Y_{t+1}^{p_{t}}=2-\beta \\
j \neq p_{t}, Y_{t}^{j} \leq q_{t} & \Longrightarrow & Y_{t+1}^{j}=Y_{t}^{j}+1, \\
j \neq p_{t}, Y_{t}^{j}>q_{t} \quad \Longrightarrow & Y_{t+1}^{j}=Y_{t}^{j} .
\end{array}
$$

We are ready to prove Theorem 1.

Proof. As $I_{t+1}^{j} \geq j$ and $Y_{t+1}^{p_{t}}=2-\beta$, we must have $\forall j \geq 2-\beta, I_{t+1}^{j} \geq Y_{t+1}^{p_{t}}$. From (14) and the assumption $p_{t^{\prime}}=p_{t}$, we must have $I_{t^{\prime}}^{p_{t}}>I_{t^{\prime}}^{p_{t}-1}$, hence $Y_{t^{\prime}}^{p_{t}}>I_{t^{\prime}}^{p_{t}-1}$ and $\forall j<p_{t}, I_{t^{\prime}}^{j}<Y_{t^{\prime}}^{p_{t}}$. Let us focus on one $j \in\left[2-\beta, p_{t}\right.$ [. There must exist a $\left.\tau \in\right] t, t^{\prime}[$ such that $I_{\tau}^{j} \geq Y_{\tau}^{p_{t}}$ and $I_{\tau+1}^{j}<Y_{\tau+1}^{p_{t}}$, an event that we call crossing. This $\tau$ must be such that $I_{\tau}^{p_{\tau}} \leq I_{\tau}^{j}$ (otherwise $I_{\tau}^{j}$ would be incremented and crossing at $\tau$ would not be possible). We prove by contradiction that in fact we must have $I_{\tau}^{j}=I_{\tau}^{p_{\tau}}$. Let us assume $I_{\tau}^{p_{\tau}}<I_{\tau}^{j}$. Consequently, $I_{\tau+1}^{j}=I_{\tau}^{j}$ and crossing at $\tau$ implies $I_{\tau}^{j}=Y_{\tau}^{p_{t}} \leq q_{\tau} \leq I_{\tau}^{p_{\tau}}$, which contradicts the assumption. Therefore, $I_{\tau}^{j}=I_{\tau}^{p_{\tau}}$. If $p_{\tau}=j$, we are done with the proof (we found a $\tau \in] t, t^{\prime}$ [ such that $p_{\tau}=j$ ). Otherwise, we have $j>p_{\tau}$ and $I_{\tau}^{j}=I_{\tau}^{j-1}=I_{\tau}^{p_{\tau}}$, hence $Y_{\tau}^{j}+1 \leq I_{\tau+1}^{j}<Y_{\tau+1}^{p_{t}} \leq Y_{\tau}^{p_{t}}+1$ (as $\left.p_{\tau} \neq p_{t}\right)$. Therefore, $Y_{\tau}^{j}<Y_{\tau}^{p_{t}}$, which implies (as $Y_{t+1}^{j} \geq Y_{t+1}^{p_{t}}$ ) that there is a $\left.\tau^{\prime} \in\right] t, \tau\left[\right.$ such that $p_{\tau^{\prime}}=j$.

Belady and Palermo noticed that the sequence of OPT distances is constrained [Belady and Palermo 1974]. In particular, they understood that no two consecutive references can have the same OPT distance if that distance is greater than two (they were assuming $\beta=0$ ). However, they did not mention Theorem 1.

Theorem 1 provides a sanity test for OPT stack implementations or algorithms emulating an OPT stack. If, by a visual inspection of the sequence of OPT distances, we find a case where Theorem 1 is violated, this is a sure indication that the algorithm or its implementation is incorrect. Note that there is no such sanity test for an LRU stack.

Corollary 1. For an OPT cache of size $j$, a time interval $\left[t, t^{\prime}\left[\right.\right.$ such that $s_{t}=s_{t^{\prime}}=s>j$ and containing $s-j+1$ cache misses must also contain at least $j-1+\beta$ cache hits.

Proof. In $\left[t, t^{\prime}\right.$ [, only $s-j$ distinct stack positions greater than $j$ can be accessed. As there are $s-j+1$ misses in $\left[t, t^{\prime}\right.$ [, by the pigeonhole principle, there must exist a 
repetition, that is, two references in $\left[t, t^{\prime}\right.$ [ at the same stack position greater than $j$. By Theorem 1 , all $j-1+\beta$ stack positions between $2-\beta$ and $j$ must be accessed between the repetition.

Corollary 2. If the number of distinct items is bounded and equal to $s$, for any $j<s$

$$
\lim _{t \rightarrow \infty} \frac{M_{[0, t[}^{j}}{t} \leq \frac{s-j}{s-1+\beta} .
$$

That is, the asymptotic miss ratio of an OPT cache of size $j$ cannot exceed $\frac{s-j}{s-1+\beta}$.

Proof. There is a miss at a finite time $t_{0}$ such that $\forall t \geq t_{0}, s_{t}=s$. The next miss occurs at $t_{1}>t_{0}$, the next one at $t_{2}>t_{1}$, and so forth. An interval $\left[t_{k(s-j)}, t_{(k+1)(s-j)}\right]$ contains exactly $s-j+1$ misses. By Corollary 1 , there must be at least $j-1+\beta$ hits in ]$t_{k(s-j)}, t_{(k+1)(s-j)}\left[\right.$. Interval $\left[t_{0}, t_{k(s-j)}\right]$ contains at least $k(j-1+\beta)$ hits. That is, $\left[t_{0}, t_{k(s-j)}\right]$ contains exactly $k(s-j)+1$ misses and at least $k(s-j)+1+k(j-1+\beta)=k(s-1+\beta)+1$ references, that is, $t_{k(s-j)}-t_{0} \geq k(s-1+\beta)$. As $k$ grows to infinity, the impact of initial misses (before $t_{0}$ ) on the overall miss ratio vanishes, and the asymptotic miss ratio does not exceed $\frac{k(s-j)}{k(s-1+\beta)}=\frac{s-j}{s-1+\beta}$.

For example, let us consider an eight-way set-associative cache $(j=8)$ managed by LRU, without bypassing $(\beta=0)$, and an application with a dataset twice larger than the cache $(s=16)$. If the measured LRU miss ratio is significantly greater than $\frac{s-j}{s-1+\beta}=\frac{16-8}{16-1} \approx 53 \%$, we do not need to run simulations with OPT to know that OPT outperforms LRU significantly on that application.

It should be noted that, for online replacement policies (such as LRU), the upper bound for the miss ratio is $100 \%$. Indeed, one can generate an ad hoc trace defeating the replacement policy, by referencing at each time $t$ an item $X_{t}$ not in the cache at time $t$. Corollary 2 implies that it is impossible to defeat OPT when the number of distinct items is bounded. The content of the OPT cache at time $t$ is not fixed, it depends on the item $X_{t}$ we are going to choose. OPT knows the future and can anticipate our moves.

\section{THE OPT MISS CURVE IS CONVEX}

Recently, Beckmann and Sanchez argued that, under optimal replacement, the miss curve must be a convex function of the cache size [Beckmann and Sanchez 2015]. They argue that, for any replacement policy with a nonconvex miss curve, it is possible, by partitioning the cache in a certain way, to derive a policy yielding fewer misses. They assume that removing some items from a trace entirely is equivalent to enlarging the cache, whatever the replacement policy. This might be approximately valid, empirically. Whether this is always true is uncertain.

With Theorem 1, we can prove rigorously that the OPT miss curve is always convex.

Corollary 3. For all $t$ and for all $j \geq 3$,

$$
M_{[0, t[}^{j-1}-M_{[0, t[}^{j} \leq M_{[0, t[}^{j-2}-M_{[0, t[}^{j-1} .
$$

Proof. The terms on the left-hand and right-hand sides of inequality (21) are, respectively, the number of references in $[0, t$ [ accessing position $j$ in the OPT stack and the number of references accessing position $j-1$. The first reference in the trace accessing position $j-1$ (i.e., when the stack size is incremented from $j-2$ to $j-1$ ) occurs before the first reference at position $j$ (when the stack size is incremented from $j-1$ to $j$ ). So (21) is true on the first access at position $j$. From Theorem 1 , between two accesses at position $j$, there must exist at least one access at position $j-1$. Therefore, on each new access at position $j$, inequality (21) remains true. 
Table IV. OPT Tokens Algorithm on an Example with Cache Size $j=3$

\begin{tabular}{|c||c|c|c|c|c|c|c|c|c|c|c|c|c|c|c|c|c|c|}
\hline$t$ & 0 & 1 & 2 & 3 & 4 & 5 & 6 & 7 & 8 & 9 & 10 & 11 & 12 & 13 & 14 & 15 & 16 & 17 \\
\hline trace & $\mathrm{A}$ & $\mathrm{B}$ & $\mathrm{C}$ & $\mathrm{D}$ & $\mathrm{E}$ & $\mathrm{F}$ & $\mathrm{A}$ & $\mathrm{B}$ & $\mathrm{C}$ & $\mathrm{D}$ & $\mathrm{E}$ & $\mathrm{F}$ & $\mathrm{A}$ & $\mathrm{B}$ & $\mathrm{C}$ & $\mathrm{D}$ & $\mathrm{E}$ & $\mathrm{F}$ \\
\hline$q_{t}$ & 1 & 2 & 3 & 4 & 5 & 6 & 6 & 6 & 6 & 6 & 6 & 6 & 6 & 6 & 6 & 6 & 6 & 6 \\
\hline \multicolumn{10}{|c|}{ without bypassing $(\boldsymbol{\beta}=\mathbf{0})$} \\
\hline$I_{t}^{3}(3)$ & 3 & 3 & 3 & 3 & 4 & 5 & 6 & 6 & 3 & 4 & 5 & 6 & 6 & 3 & 4 & 5 & 6 & 6 \\
\hline$I_{t}^{3}(2)$ & 2 & 2 & 2 & 2 & 3 & 4 & 5 & 2 & 2 & 3 & 4 & 5 & 2 & 2 & 3 & 4 & 5 & 2 \\
\hline$I_{t}^{3}(1)$ & 1 & 1 & 1 & 1 & 1 & 1 & 1 & 1 & 1 & 1 & 1 & 1 & 1 & 1 & 1 & 1 & 1 & 1 \\
\hline miss & & & & $\mathrm{m}$ & $\mathrm{m}$ & $\mathrm{m}$ & & & $\mathrm{m}$ & $\mathrm{m}$ & $\mathrm{m}$ & & & $\mathrm{m}$ & $\mathrm{m}$ & $\mathrm{m}$ & & \\
\hline \multicolumn{10}{|c||}{} & & \multicolumn{10}{|c|}{ with bypassing $(\boldsymbol{\beta}=\mathbf{1})$} & & & & \\
\hline$I_{t}^{3}(3)$ & 3 & 3 & 3 & 3 & 4 & 5 & 6 & 6 & 6 & 3 & 4 & 5 & 6 & 6 & 6 & 3 & 4 & 5 \\
\hline$I_{t}^{3}(2)$ & 2 & 2 & 2 & 2 & 3 & 4 & 5 & 5 & 2 & 2 & 3 & 4 & 5 & 5 & 2 & 2 & 3 & 4 \\
\hline$I_{t}^{3}(1)$ & 1 & 1 & 1 & 1 & 2 & 3 & 4 & 1 & 1 & 1 & 2 & 3 & 4 & 1 & 1 & 1 & 2 & 3 \\
\hline miss & & & & $\mathrm{m}$ & $\mathrm{m}$ & $\mathrm{m}$ & & & & $\mathrm{m}$ & $\mathrm{m}$ & $\mathrm{m}$ & & & & $\mathrm{m}$ & $\mathrm{m}$ & $\mathrm{m}$ \\
\hline
\end{tabular}

As noted by Beckmann and Sanchez, if a replacement policy's miss curve is markedly nonconvex, it is certainly far from the OPT miss curve [Beckmann and Sanchez 2015].

\section{THE OPT TOKENS ALGORITHM}

The OPT matrix introduced in Section 8 considers all cache sizes simultaneously. When we focus on a single cache size, a simpler algorithm can be derived from the OPT matrix.

Notice that, for a fixed $j$, the $s_{t}$ values $D_{t}^{j}(i)$ for $i \in\left[1, s_{t}\right]$ are sufficient to determine hits and misses in a cache of size $j$. In practice, we only need the values $D_{t}^{j}(i) \leq j$. Moreover, there are only $j$ such distinct values. Let us generalize quantity $I_{t}^{j}$ as follows:

$$
\text { for } i \in[1, j], \quad I_{t}^{j}(i):=\min \left\{k \in\left[1, s_{t}\right]: D_{t}^{j}(k)=i\right\} \text {. }
$$

We have $I_{t}^{j}(j)=I_{t}^{j}$. Note that $I_{t}^{j}(i)<I_{t}^{j}(i+1)$ (cf. definition (17)). From the update procedure (20), we obtain what we call the OPT tokens algorithm:

\begin{tabular}{|c|ll|}
\hline Case & \multicolumn{1}{|c|}{ Action } \\
\hline \hline start & & $I_{0}^{j}(i)=i$ \\
\hline$q_{t}>I_{t}^{j}(j)$ & $\beta=0$ & $I_{t+1}^{j}(1)=1$ \\
miss & $i \geq 2-\beta$ & $I_{t+1}^{j}(i)=I_{t}^{j}(i)+1$ \\
\hline$q_{t} \leq I_{t}^{j}(j)$ & & $I_{t+1}^{j}(1)=1$ \\
$h i t$ & $I_{t}^{j}(i)<q_{t}$ & $I_{t+1}^{j}(i+1)=I_{t}^{j}(i)+1$ \\
& $I_{t}^{j}(i) \geq q_{t}$ & $I_{t+1}^{j}(i+1)=I_{t}^{j}(i+1)$ \\
\hline
\end{tabular}

The OPT tokens algorithm takes as input the LRU stack positions $\left(q_{t}\right)$ generated from the input trace $\left(X_{t}\right)$. Consistent with our choice to define $q_{t}=s_{t}+1$ upon referencing an item for the first time, the first reference to an item occurring at a time $t$ such that $s_{t}<j$ is counted as a hit. Misses can occur only when $s_{t} \geq j$. Table IV shows how the OPT tokens algorithm works on an example with six distinct items referenced in a circular fashion, assuming cache size $j=3$.

Figure 4 shows a visual for the OPT tokens. The number of tokens is equal to the cache size $j . I_{t}^{j}(i)$ is the height of the $i^{t h}$ token. An OPT miss occurs if $q_{t}$ is above the highest token, that is, $I_{t}^{j}(j)$. Otherwise, this is an OPT hit. Then, the tokens are moved according to the rules (22). 


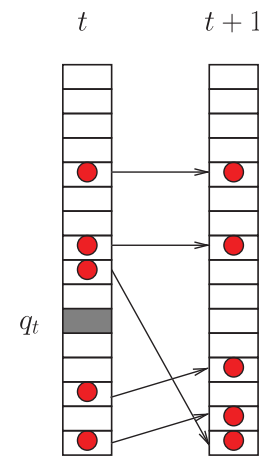

hit

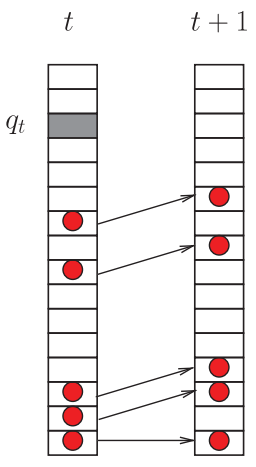

miss (no bypass)

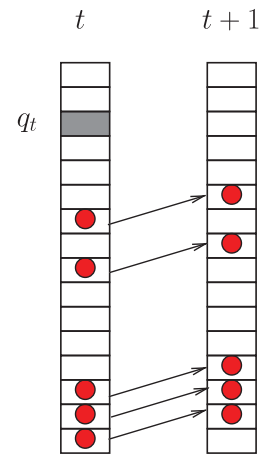

miss (bypass)

Fig. 4. Visual for the OPT tokens. The number of tokens is equal to the cache size (here 5). If $q_{t}$ (the LRU stack position of item $X_{t}$ ) is above the highest token, this is an OPT miss, otherwise this is an OPT hit.

\section{CACHE BYPASSING}

Historical papers about optimal replacement studied paging in main memory [Belady 1966; Mattson et al. 1970; Belady and Palermo 1974]. They considered the caching problem without bypassing the cache (which is main memory here). To the best of our knowledge, Scott McFarling was the first to consider optimal replacement with bypassing [McFarling 1991]. McFarling modified the OPT policy as shown in Figure 2. Let us denote OPTb and OPTn the OPT policy with and without bypassing, respectively.

We are not aware of any published proof that OPTb is optimal. ${ }^{5}$ It is relatively straightforward, however, to take one of the several published optimality proofs for OPTn, for example, Lee et al. [2016], and to adapt it to prove the optimality of OPTb. We will not do this here.

In his thesis, McFarling proved the following:

McFarling's inequality. An OPTb cache of size $j$ generates no more misses than an OPTn cache of size $j$ and no more hits than an OPTn cache of size $j+1$.

McFarling's argument is very simple. An OPTn cache cannot generate fewer misses than an equal-sized OPTb cache, as OPTb is optimal and outperforms all policies, including those like OPTn that never bypass the cache. For the other part of the inequality, McFarling gives the following argument. If one takes an OPTb cache of size $j$ and stores in a victim cache of size 1 the item evicted from the OPTb cache or bypassing the OPTb cache, the OPTb cache and the victim cache together can be viewed as a cache of size $j+1$ that is not bypassed. Hence, the OPTb+victim cache (and the OPTb cache alone) does not generate more hits than an OPTn cache of size $j+1$.

The OPT tokens algorithm provides another way to relate OPTn and OPTb. Upon an OPTn miss (see Figure 4), all the tokens but the lowest one move upwards. The lowest token never leaves the bottom position. Apart from that, OPTn and OPTb behave identically. More precisely, if we ignore hits at $q_{t}=1$, an OPTn cache of size $j+1$ behaves like an OPTb cache of size $j$ seeing reuse distances $q_{t}^{\prime}=q_{t}-1$. That is, the two caches yield exactly the same hits and misses. This equivalence is summarized in Figure 5.

For example, consider a random trace with $s$ distinct items, that is, such that all LRU stack positions in $[1, s]$ have equal probability $1 / s$ to be accessed. Let us denote

\footnotetext{
${ }^{5} \mathrm{McF}$ arling did not provide an actual proof that OPTb is optimal.
} 


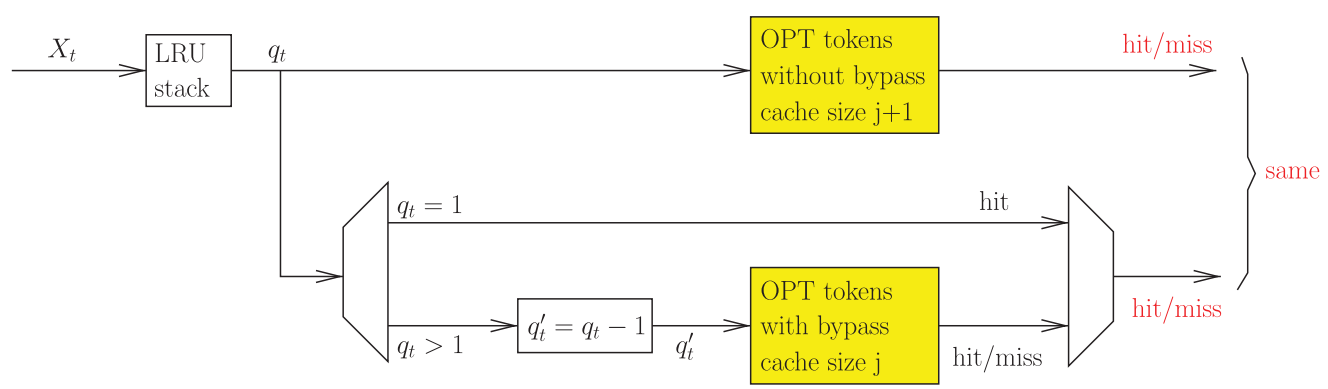

Fig. 5. Equivalence between an OPT cache of size $j+1$ without bypassing and an OPT cache of size $j$ with bypassing.

$m_{n}(j, s)$ and $m_{b}(j, s)$ the OPT miss ratios for an OPT cache of size $j$ on random traces, without and with bypassing, respectively. We mentioned in Section 7 that there is probably no simple mathematical formula for computing exactly $m_{n}$ and $m_{b}$. Yet, from the equivalence of Figure 5, we can derive a simple equation relating $m_{n}$ and $m_{b}$. A fraction $1 / s$ of references are such that $q_{t}=1$ (hit). After applying the transformation shown in Figure 5, we obtain a random trace such that all LRU stack positions in $[1, s-1]$ have equal probability to be accessed. This yields the following equality:

$$
m_{n}(j+1, s)=\left(1-\frac{1}{s}\right) \times m_{b}(j, s-1) .
$$

\section{CIRCULAR TRACES}

We already mentioned that, if the number $s$ of distinct items is bounded, OPT cannot be defeated, that is, its miss ratio is always below 100\% (Corollary 2). On the other hand, all online replacement policies can be defeated. In particular, it is well known that, when the cache size $j$ is less than $s$, accessing $s$ distinct items in a circular fashion (as in the example of Table IV) defeats LRU.

The OPT miss ratio on a circular trace can be calculated easily (see, for instance, Smith and Goodman [1985] for an OPT cache without bypassing). One can check that the OPT tokens algorithm yields the correct result (see Table IV). For OPT with bypassing, the steady-state miss ratio on a circular trace is

$$
\text { OPTb miss ratio on a circular trace }=\frac{s-j}{s} .
$$

For OPT without bypassing, we replace $j$ by $j-1$ and $s$ by $s-1$ in the preceding expression (cf. Figure 5) and we obtain $\frac{(s-1)-(j-1)}{s-1}$, that is,

$$
\text { OPTn miss ratio on a circular trace }=\frac{s-j}{s-1} .
$$

The steady-state OPT miss ratio is equal to the upper bound stated by Corollary 2. In other words, accessing a dataset in a circular fashion maximizes the OPT miss ratio.

It is not coincidental that the trace with the worst OPT miss ratio is the one that maximizes $q_{t}$. We are going to show the following:

Proposition 1. If two traces $\mathcal{T}=\left(X_{t}\right)$ and $\mathcal{T}^{\prime}=\left(X_{t}^{\prime}\right)$, generating reuse distances $\left(q_{t}\right)$ and $\left(q_{t}^{\prime}\right)$, respectively, have the same length and are such that $q_{t} \leq q_{t}^{\prime}$ for all $t$, then $\mathcal{T}$ does not generate more OPT misses than $\mathcal{T}^{\prime}$.

Proof. To show Proposition 1, it is sufficient to start from a trace $\left(q_{t}\right)$, increment one $q_{\tau}\left(q_{\tau}^{\prime}=q_{\tau}+1, q_{t}^{\prime}=q_{t}\right.$ for $\left.t \neq \tau\right)$, and show that the number of misses does not 

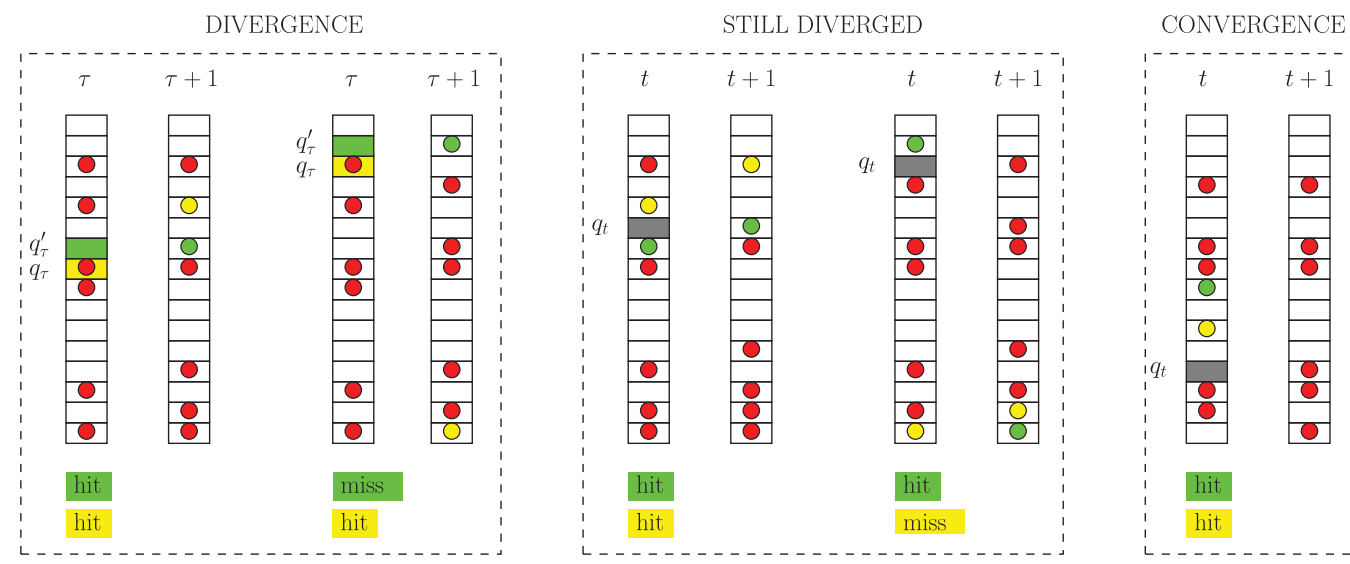

Fig. 6. The different cases for proving Proposition 1. At time $\tau$, trace $\mathcal{T}$ accesses LRU stack position $q_{\tau}$, and trace $\mathcal{T}^{\prime}$ accesses LRU stack position $q_{\tau}^{\prime}=q_{\tau}+1$. For $t>\tau$, the two traces access the same LRU stack positions. The diverging token for trace $\mathcal{T}$ is colored in yellow, and the diverging token for $\mathcal{T}^{\prime}$ in green (the diverging token for a trace is not a token for the other trace; they are shown simultaneously for the sake of succinctness). The "leader" trace is the one whose diverging token is the lowest. When a miss for the leader trace is a hit for the other trace, the leader changes.

decrease. Figure 6 provides examples illustrating the different cases. Before time $\tau$, the two traces have exactly the same behavior. If $q_{\tau}$ does not coincide with a token, no divergence occurs, and the two traces continue to have the same behavior. Otherwise, if $q_{\tau}$ coincides with a token, the two traces diverge. Once diverged, the two sets of tokens (one set for each trace) coincide except for one token, the diverging token. In Figure 6, the two diverging tokens (one for each trace) are shown simultaneously; the one for trace $\mathcal{T}$ colored in yellow, and the one for trace $\mathcal{T}^{\prime}$ colored in green. We distinguish two situations. We say that trace $\mathcal{T}$ is the "leader" if its diverging token is lower than the diverging token of trace $\mathcal{T}^{\prime}$. Otherwise, trace $\mathcal{T}^{\prime}$ is the leader.

If there is divergence at $\tau$, two situations can occur (left part of Figure 6):

(1) both $\mathcal{T}$ and $\mathcal{T}^{\prime}$ hit at $\tau: \mathcal{T}^{\prime}$ is the leader at $\tau+1$.

(2) $\mathcal{T}$ hits and $\mathcal{T}^{\prime}$ misses: $\mathcal{T}$ is the leader at $\tau+1$.

For $t>\tau$ and before convergence, two situations can occur (middle part of Figure 6):

(1) $\mathcal{T}$ and $\mathcal{T}^{\prime}$ both hit or both miss at $t$ : the leader at $t$ is the leader at $t+1$.

(2) The leader trace misses and the other trace hits: the leader changes.

Finally, convergence can occur only when both traces hit (right part of Figure 6). After convergence, the two sets of tokens coincide, and the two traces have exactly the same behavior.

Between divergence and convergence, for having a miss for $\mathcal{T}$ and a hit for $\mathcal{T}^{\prime}$ at $t$, the leader at $t$ must be $\mathcal{T}$. But if $\mathcal{T}$ is the leader at $t$, it means that there was previously a hit for $\mathcal{T}$ and a miss for $\mathcal{T}^{\prime}$. Hence, $\mathcal{T}$ does not generate more misses than $\mathcal{T}^{\prime}$.

From Proposition 1, it is obvious why circular accesses are worst for OPT. Sometimes, Proposition 1 can also provide a lower bound for the number of OPT misses:

Corollary 4. For an OPT cache of size $j$, on an infinitely long trace, if there exists a finite time $\tau$ such that for all $t \geq \tau, q_{t} \geq q_{\min }>j$, the asymptotic OPT miss ratio is greater than or equal to $\frac{q_{\min }-j}{q_{\min }-1+\beta}$. 
Proof. We transform trace $\left(q_{t}\right)$ into $\left(q_{t}^{\prime}\right)$ such that $q_{t}^{\prime}=q_{t}$ for $t<\tau$ and $q_{t}^{\prime}=q_{\text {min }}$ for $t \geq \tau$. From Proposition 1, the OPT miss ratio of $\left(q_{t}\right)$ is greater than or equal to that of $\left(q_{t}^{\prime}\right)$. For $t \geq \tau$, trace $\left(q_{t}^{\prime}\right)$ has a circular behavior (i.e., $q_{t}^{\prime}$ is constant). Whatever the positions of tokens at $\tau$, the asymptotic miss ratio of $\left(q_{t}^{\prime}\right)$ is $\frac{q_{\min }-j}{q_{\min }-1+\beta}$.

If a trace is such that $q_{t}$ is not constant but remains in an interval $\left[q_{\min }, q_{\max }\right]$, the asymptotic OPT miss ratio is in the interval $\left[\frac{q_{\min }-j}{q_{\min }-1+\beta}, \frac{q_{\max }-j}{q_{\max }-1+\beta}\right]$. If $q_{\min }$ and $q_{\max }$ are close, we know approximately the OPT miss ratio.

\section{CONCLUSION}

Belady's MIN algorithm and Mattson's OPT replacement policy provide two different ways to think about optimal replacement. This article brings three new equivalent ways to reason about OPT hits and misses: Equation (14), the OPT matrix, and the OPT tokens. With these conceptual tools, we have discovered and proved several facts that were previously unknown, and we have proved rigorously the convexity of OPT miss curves. Theorem 1 highlights a remarkable structure in traces of OPT distances, and offers a very simple way to detect bugs in OPT stack implementations, just by a visual inspection of the output. Also, we found that the notion of reuse distance, which is naturally related to the LRU policy, is also useful for understanding optimal replacement. Probably, many mathematical facts about optimal replacement remain to be discovered.

Though the goal of this article is to improve our understanding of optimal replacement, some of our results might lead to practical findings. For instance, the Hawkeye cache replacement policy is directly inspired from Belady's MIN algorithm [Jain and Lin 2016]. The OPT tokens algorithm might inspire alternate implementations of the Hawkeye policy.

\section{REFERENCES}

A. V. Aho, P. J. Denning, and J. D. Ullman. 1971. Principles of optimal page replacement. Journal of the ACM 18, 1 (Jan. 1971).

N. Beckmann and D. Sanchez. 2015. Talus: A simple way to remove cliffs in cache performance. In International Symposium on High Performance Computer Architecture (HPCA).

L. A. Belady. 1966. A study of replacement algorithms for virtual-storage computers. IBM Systems Journal $5,2(1966)$.

L. A. Belady and F. P. Palermo. 1974. On-line measurement of paging behavior by the multivalued MIN algorithm. IBM Journal of Research and Development 18, 1 (Jan. 1974).

J. Brock, X. Gu, B. Bao, and C. Ding. 2013. Pacman: Program-assisted cache management. In International Symposium on Memory Management (ISMM).

D. Burger and T. M. Austin. 1997. The simplescalar tool set, version 2.0. ACM SIGARCH Computer Architecture News 25, 3 (June 1997).

E. G. Coffman and P. J. Denning. 1973. Operating Systems Theory. Prentice-Hall.

A. Cohen and W. A. Burkhard. 1995. A proof of the optimality of the MIN paging algorithm using linear programming duality. Operations Research Letters 18, 1 (Aug. 1995).

A. Jain and C. Lin. 2016. Back to the future: Leveraging Belady's algorithm for improved cache replacement. In International Symposium on Computer Architecture (ISCA).

D. E. Knuth. 1985. An analysis of optimum caching. Journal of Algorithms 6, 2 (June 1985).

M.-K. Lee, P. Michaud, J. S. Sim, and D. Nyang. 2016. A simple proof of optimality for the MIN cache replacement policy. Information Processing. Letters 116, 2 (Feb. 2016).

W.-F. Lin and S. Reinhardt. 2002. Predicting Last-Touch References Under Optimal Replacement. Technical Report CSE-TR-447-02. University of Michigan.

R. L. Mattson, J. Gecsei, D. R. Slutz, and I. L. Traiger. 1970. Evaluation techniques for storage hierarchies. IBM Systems Journal 9, 2 (1970).

S. McFarling. 1991. Program Analysis And Optimization For Machines With Instruction Cache. Ph.D. dissertation. Stanford University. 
T. R. Puzak. 1985. Analysis of Cache Replacement Algorithms. Ph.D. dissertation. University of Massachusetts Amherst.

K. Rajan and R. Govindarajan. 2007. Emulating optimal replacement with a shepherd cache. In International Symposium on Microarchitecture (MICRO).

A. J. Smith. 1976. Analysis of the optimal, look-ahead demand paging algorithms. SIAM Journal on Computing 5, 4 (Dec. 1976).

J. E. Smith and J. R. Goodman. 1985. Instruction cache replacement policies and organizations. IEEE Transactions on Computers C-34, 3 (March 1985).

R. A. Sugumar and S. G. Abraham. 1993. Efficient simulation of caches under optimal replacement with applications to miss characterization. In ACM SIGMETRICS.

O. Temam. 1999. An algorithm for optimally exploiting spatial and temporal locality in upper memory levels. IEEE Transactions on Computers 48, 2 (Feb. 1999).

B. Van Roy. 2007. A short proof of optimality for the MIN cache replacement algorithm. Information Processing Letters 102, 2 (April 2007).

W. Vogler. 2008. Another short proof of optimality for the MIN cache replacement algorithm. Information Processing Letters 106, 5 (May 2008).

Received August 2016; revised November 2016; accepted November 2016 